\title{
Imaging Surface Plasma of Metals by Ultrafast Transmission Electron Microscopy
}

Zhiwei Wang ${ }^{1}$ and Deshuai $\mathrm{Li}^{1}$

1. Beijing Institute of Nanoenergy and Nanosystems, Chinese Academy of Sciences, Beijing, P. R. China.

Tremendous progresses have been made in developing abreaction-correction techniques in transmission electron microscopy, which enables direct imaging of individual atoms and atomic defects routinely [12]. However, owing to inherent speed limitation of image recording devices, temporal resolution achieved in conventional TEM has been mainly restricted to millisecond level. Time-resolved transmission electron microscopy was thus developed to investigate ultrafast dynamic processes such as atomic motions, acoustic resonances, and structural phase transitions etc., through a combination of TEM and ultrashort pulsed lasers for pump-probe photoelectron pulse imaging [3-4].

Surface plasma (collective electron oscillations) of metals has been widely explored for both the fundamental interest and their practical applications in molecular sensors, and catalysis etc. Ultrafast electron microscopy is an ideal tool for studying the surface plasmon phenomenon, but previous researches on this aspect appeared to mainly focus on time-zero determination of probe-pump optical path through exiting $\mathrm{Cu}$ TEM grids [5]. Based on a recently built UTEM, we carried out a quantitative investigation of surface plasma of metal surfaces for identifying the effect of the probe and pump laser beam fluences, materials, and surface morphologies.

The experimental investigation was performed on a 200kV FEI Tecnai UEM integrated with a Clark Impulse fiber laser with pulse duration of $\sim 250 \mathrm{fs}$ and average power of $20 \mathrm{~W}$. To realize pump-probe imaging, an ultrafast pulse ( $\sim 1.03$ um center wavelength) out of the Impulse laser is split into two beams after frequency doubling. One is used to pump the specimen (connecting with a linear optical delay stage), and the other is directed to a $150 \mathrm{um} \mathrm{LaB}_{6}$ photocathode inside electron microscope after frequency quadrupling for producing ultrashort photoelectron packets. Commercially available $\mathrm{Cu}$ and $\mathrm{Au}$ TEM grids are directly used in the surface plasmon experiments. Time-delay UTEM images were generally recorded at low-mag mode $(330 \mathrm{x})$ to provide high SNRs for data analysis. This is especially useful for weak electron packet imaging as the method of increasing exposure time for SNR enhancement may also result in blurred images. Intensity profiling and sigmoidal fitting analysis was performed for accurately determining the position distribution of surface plasma/electron clouds formed by the laser excitation [6-7].

Figure 1 displays time-evolution dynamics of surface plasma extracted from pump-probe UTEM imaging of $\mathrm{Cu}$ TEM girds (2000 meshes) at basically the same pump fluence $(\sim 11.7 \mathrm{mj} / \mathrm{cm} 2)$. These plots were made by averaging all the separations from four different regions ("A"-"D" in Figure 1a inset) around the laser beam center "o". The figures illustrate clearly that the formation and timeevolution process of $\mathrm{Cu}$ surface plasma occurs within an ultrashort time period. When imaging by a comparatively weak photoelectron packet $(0.2 \mathrm{~mW}$, measured at the column entrance), we observed a sharp rise of surface plasmon edge (Figure 1a). By employing an error function to fit the formation process of surface plasma, a response time constant of $\sigma=0.45 \mathrm{ps}$ was obtained ( $\sigma$ denotes standard deviation of the Gaussian fitting), as shown in Figure 1b. When higher electron packet intensities were used for imaging (Figure 1c-1d), significant increase of time constants was observed due to to spacecharge broadening effect. Given the laser pulse duration of $250 \mathrm{fs}$ used in this work, it is expected that 
the even smaller time constant $(<0.45 \mathrm{ps})$ can be achieved by decreasing photoelectron packet intensities further.

The surface plasmon dynamics of metal grids can also be affected by pump beam fluence and specimen morphologies etc., as demonstrated in our ongoing study, the details of which will be discussed in the future. In general, the study indicates that, by selecting/refining laser excitation and specimen morphology conditions, surface plasma of metals could be generated in a desired way. The tunable surface plasma may also serve a purpose of spatially manipulating the incident photoelectron pulses with ultrafast temporal controllability.

\section{References:}

[1] YAR Dasilva et al, Ultramicroscopy 176 (2017), p.11.

[2] ZW Wang and RE Palmer, Nano Lett 12 (2002), p.91.

[3] AH Zewail, Science 328 (2010), p.187.

[4] DJ Flannigan and AH Zewail, Acc Chem Res 45 (2012), p.1828.

[5] GL Cao et al, Sci Rep 5 (2015), p.8404.

[6] DA Plemmons and DJ Flannigan, Chem. Phys. Lett 683 (2017), p186.

[7] DR Cremons, PhD thesis, University of Minnesota (2017).
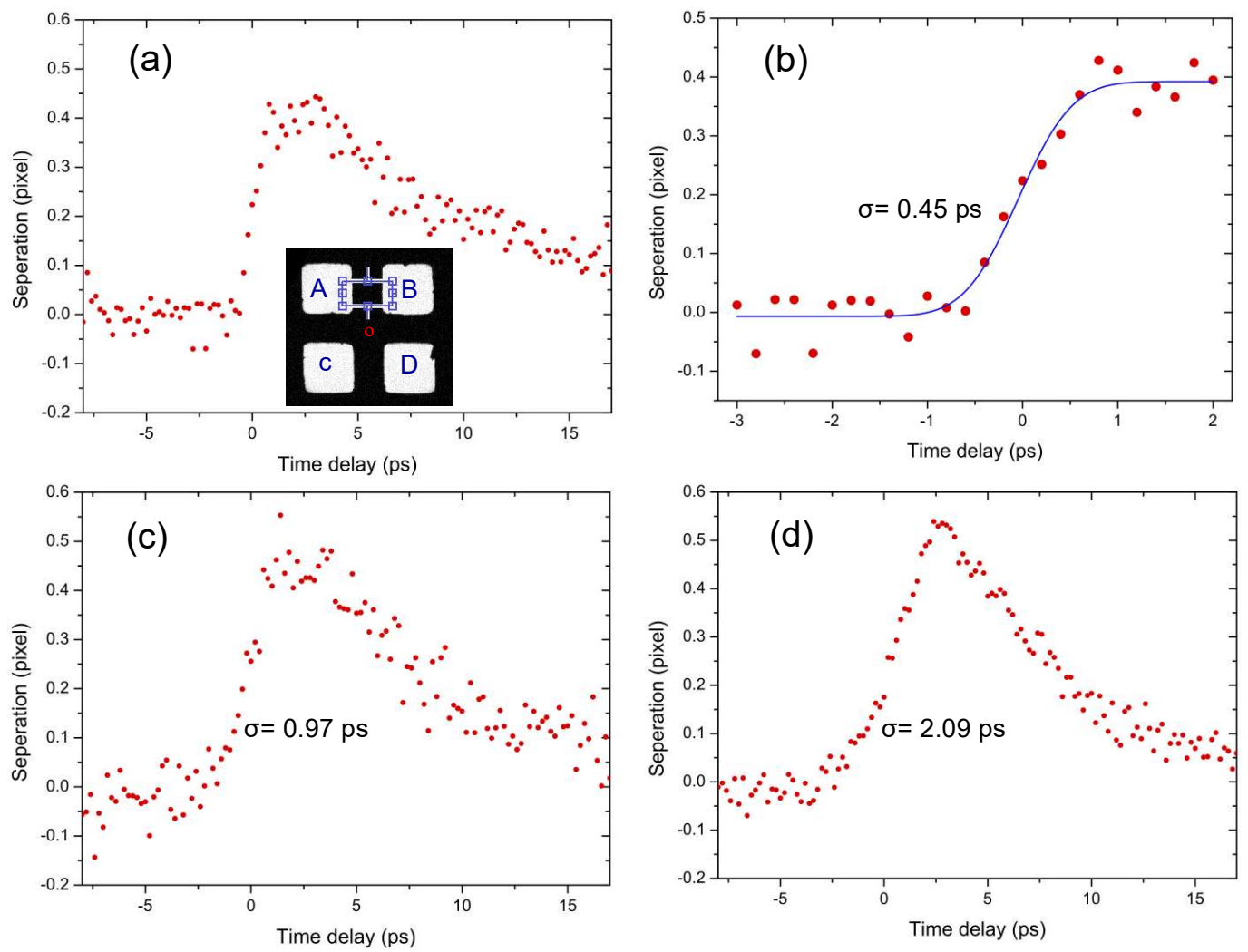

Figure 1. Effect of photoelectron beam intensity on the dynamics of surface plasma formed on $\mathrm{Cu}$ TEM grids. (a), (c) and (d) are acquired with photoelectron packet intensities of 0.2, 1 and $5 \mathrm{~mW}$ (measured at the microscope column entrance), respectively. (b) is a enlarged view of (a) at the formation stage of surface plasma. The inset to (a) shows a low-mag TEM image of $\mathrm{Cu}$ grids, in which the region marked with "o" denotes the central peak position of pump laser beam. 